\title{
XXI.
}

\section{Ueber den Einfluss verschiedener Salze auf die Entwickelung der Zuckerrübe.}

\author{
Von \\ Dr. G. Horth.
}

Die grosse Bedeutung, welche die Runkelrübe sowohl in industrieller als landwirthschaftlicher Beziehung erreicht hat, muss das Interesse des landwirthschaftlichen Chemikers um so mehr in Anspruch nehmen, als die Rübencultur gegenüber den bereits in der Rübenzucker- und Alkoholfabrikation gelösten technischen Schwierigkeiten, noch eines jeden rationellen Verfahrens entbehrt, und doch werden diese so wichtigen Industriezweige so lange in ihrer Existenz bedroht und gefährdet sein, als die RübenCultur einer wissenschaftlichen Grundlage entbehrt.

Mit der Beantwortung der Frage: Welchen Einfluss üben die Bodenbeschaffenheit, die verschiedenen Düngungsmittel, das Klima, die Aufbewahrungsart etc. auf die chemische Beschaffenheit der Rüben aus, wird auch die Frage beantwortet sein, ob diese beiden jungen, aber bereits mächtig dastehenden Industriezweige eine Zukunft haben oder nicht.

Ich habe daher in Nachstehendem die Frage zu beantworten versucht: Welchen Einfluss die verschiedenen Salze (als Düngungsmittel) auf die Erträge und auf die chemische Beschaffenheit, besonders den Zucker- und Stickstoffgehalt der Rüben ausüben.

Das Versuchsfeld war ein thonhaltiger humusreicher Sandboden, welcher im vorhergehenden Jahre ohne Dünger mit Tabak bestellt, nun ohne allen Dünger zur Aufnahme der Rübenpflanzen hergerichtet wurde.

Die Versuchsbeete I. bis XIII. zerfielen jedes in kleinere Abtheilungen von je 16 Q.-Fuss, um nicht allein den Einfluss der verschiedenen Salze, sondern auch den Einfluss der verschiedenen Quantitäten der aufgebrachten Salze Journ. t. prakt Chemie. LXIV. 3. 
ermitteln zu können. Die einzelnen Abtheilungen wurden durch 1 Fuss breite festgestampfte Rinnen von einander getrennt.

Die Pflanzen (weisse schlesische Rübe) wurden aus dem hiesigen landwirthschaftlichen Garten bezogen und Anfang Juni auf je 16 Q.-Fuss 8 Pflanzen gesetzt.

Die zu den Versuchen. dienenden Salze wurden bei $100^{\circ}$ getrocknet und gleichförmig auf das ganze Versuchsbeet ausgestreut mit dem Boden vermischt.

Mit Ausnahme der mit Kochsalz gedüngten Pflanzen (welche anfangs zu leiden schienen, sich aber sehr bald wieder erholten) ging die Vegetation so normal von Statten, wie es bei dem, viele Monate hindurch anhaltenden regnerischen Wetter nur immer $\mathrm{zu}$ erwarten war.

Unter allen zeichneten sich die mit Ammoniaksalzen, kohlensaurem Kali, Kali und Natron-Salpeter gedüngten Pflanzen durch ihre Ueppigkeit und sattgrüne Farbe der Blätter aus.

Mit der Ernte musste (wegen eintretender Fröste) schon am 24. October begonnen werden. Die lockere Beschaffenheit des Bodens liess die Rüben sehr leicht mit der Hand herausziehen.

Die Rüben, an der Blattbasis von den Blättern befreit, wurden gewaschen, abgetrocknet und sogleich gewogen, ergaben folgende Erträge:

Auf 16 Q.-Fuss.

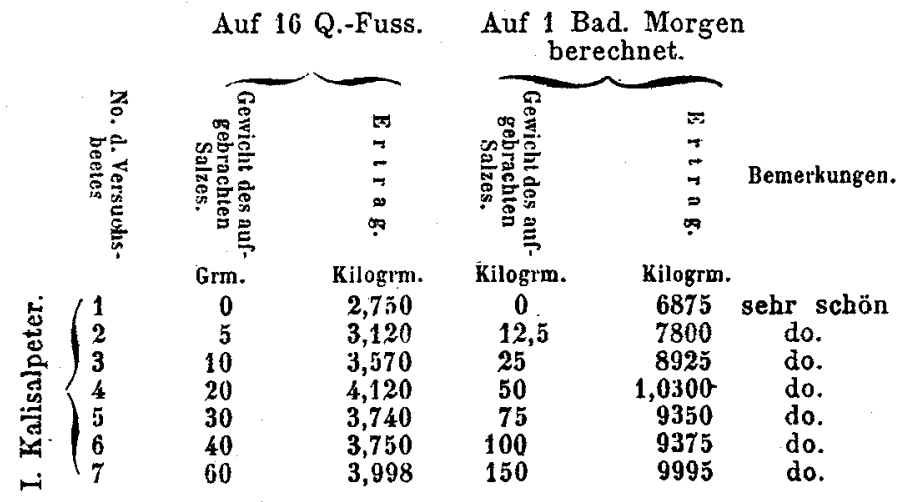


Auf 16 Q.-Fuss. Auf 1 Bad. Morgen

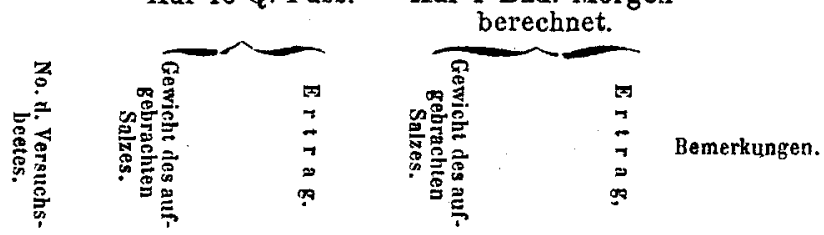

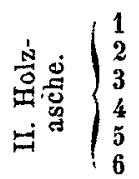

Grm.

Kilogrnn

Kilogrm.

Kilogrm.

$0 \quad 2,750$

$10 \quad 2,374$

$20 \quad 3,642$

$\mathbf{2 5}$

$\mathbf{3 , 6 4 2} \quad \mathbf{5 0}$

40

3,751

50

$3,512, \quad 125$

$3,250 \quad 150$

6875 schön

5935 etwas hell

910 schön

9377 do.

60

3,786

造葛 $\left\{\begin{array}{l}1 \\ 2 \\ 3 \\ 4 \\ 5 \\ 6\end{array}\right.$

0
10

3,140

0

8780 do.

8140 do.

15

30

50

4,250

4,506

5,106

25

946 schōn

7850 do.

1,0625 do.

$1,126 \mathrm{j}$ do.

75

$\mathbf{5 , 6 1 0}$

1,2762

1,4025

do.

150

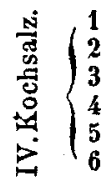

2,560

3,254

0

3,120

2,501

12,5

6400 etwas holzig

8135 do.

7800 do.

6252 do.

8082

8765

do.

$\mathbf{3}, 507 \quad 200$

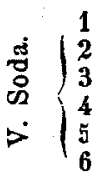

2,258

2,510

2,161

2,120

2,527

3,019

\section{0}

25

15
30

50

37

75

120

125

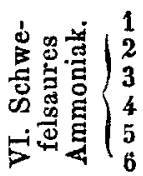

2,512

$2,7,50$

0

3,256

12

2,320

25

3,561

37

3,214

63

25
40

100

离总焉 $\left\{\begin{array}{l}1 \\ 2 \\ 3\end{array}\right.$

3,250

4,375

0
12

3,736

4,502

光离易 5

3,267

25

37

15
25

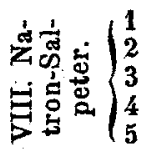

$\begin{array}{rrrrc}0 & \mathbf{4}, 210 & 0 & 1,0525 & \text { sehr schōn } \\ 20 & \mathbf{5 , 5 6 3} & \mathbf{5 0} & \mathbf{1 , 3 9 0 7} & \text { do. } \\ \mathbf{3 0} & \mathbf{4 , 9 6 5} & \mathbf{7 5} & \mathbf{1} 2412 & \text { do. } \\ 40 & \mathbf{5 , 1 1 4} & \mathbf{1 0 0} & 1,2785 & \text { do. } \\ \mathbf{6 0} & \mathbf{6 , 0 3 1} & \mathbf{1 5 0} & \mathbf{1 , 5 0 7 7} & \text { do. }\end{array}$

5645 holzig

6275 do.

5402 do.

5300 do.

6317 do.

7547 do.

6280 schön

6875 do.

8140 do.

5800 etwas bell

8902

8035

do.

8125 schön

1,0937 do.

9340 do.

1,1405 do.

8167 do. 


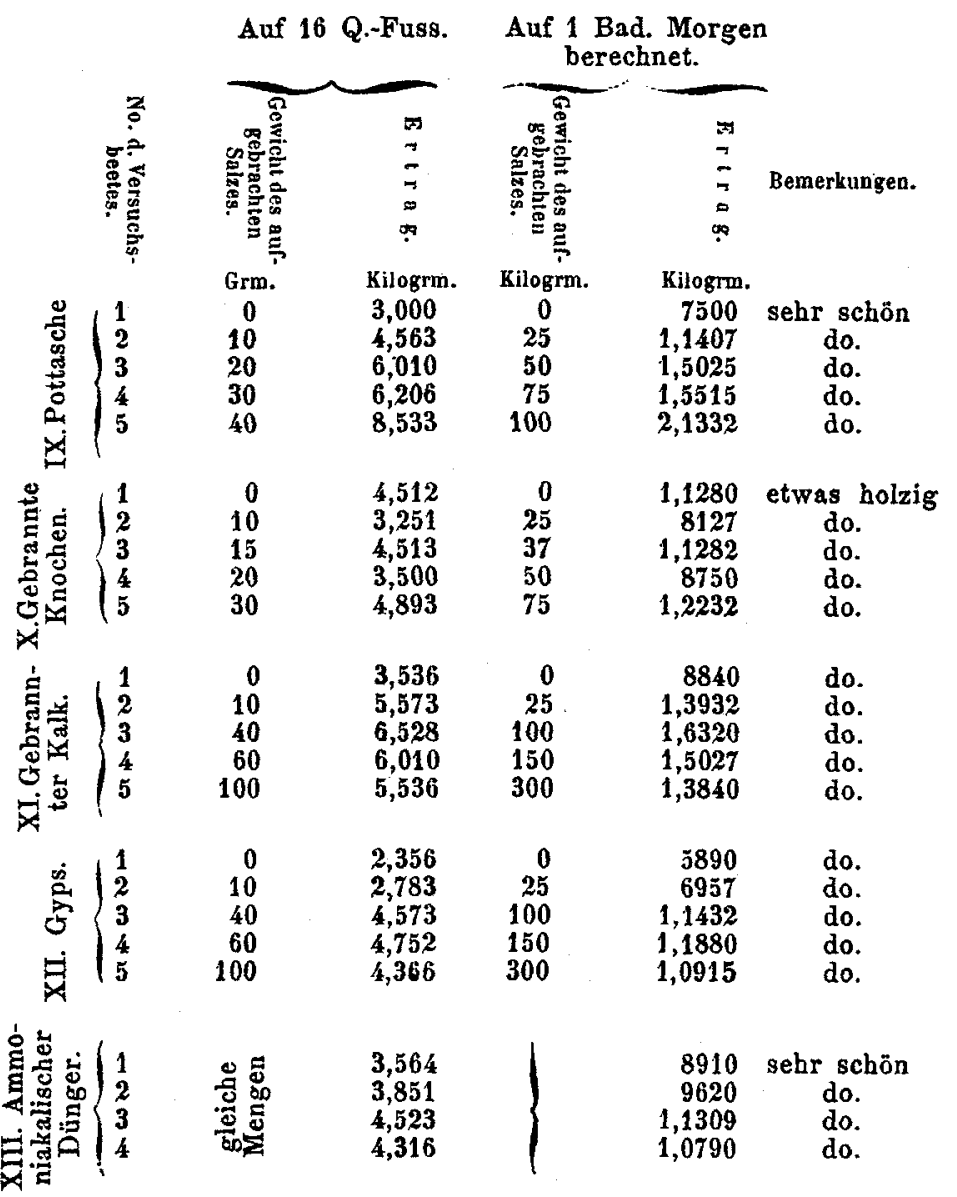

Die grossen Schwankungen in den ungedüngten Beeten liegen theils in der Natur der Rüben, theils mag ihnen auch durch Ueberschwemmungen von den Salzen der benachbarten Beete zugeführt worden sein, wohin auch die nachfolgende quantitative Analyse deutet.

Um nun den Einfluss der so verschiedenen Salze auf die chemische Beschaffenheit der Rüben zu untersuchen, wurden zur Analyse möglichst gleich grosse Rüben ausgesucht und von diesen ein Querschnitt des mittleren Rübentheils zur Untersuchung verwendet. 
Was die Versuchsmethode angeht, so wurde bei allen der gleiche Gang eingehalten.

Zur Bestimmung des Wassers wurden die Rüben in lange dünne Scheiben geschnitten und an Fäden frei hängend zuerst in einem geheizten Raum, und dann erst in mit Deckel versehenen Porcellanschalen bei $100^{\circ} \mathrm{im}$ Wasserbad getrocknet. Das Trocknen geht auf diese Weise sehr rasch, und die Rübenscheiben behalten ihre weisse Farbe. Das Einäschern geschah im Platintiegel.

Die Bestimmung des Zuckergehaltes wurde nach vielen andern versuchten Methoden auf folgende Weise ausgeführt.

Ohngefähr 50 bis $100 \mathrm{Grm}$. Rüben wurden auf dem Reibeisen zerrieben und nun mit dem 6 fachen Gewichte schwefelsäurehaltigen Wassers eine halbe Stunde hindurch gekocht, und nach dem Erkalten der so verdünnte Rübenbrei in einem $1 \frac{1}{2}$ Fuss langen länglichen, unten mit einem Baumwollenpfropf versehenen Retortenvorstoss filtrirt und so lange ausgewaschen, bis eine abfiltrirte Probe auf Kupfertartrat nicht mehr reagirte, was gewöhnlich nach 3- bis 4-maligem Aufgiessen mit frischem Wasser der Fall ist. Dieses so erhaltene Filtrat kann, obwohl immer etwas opalisirend, ohne weitere Klärung zur Zuckerbestimmung mittels der Fehling'schen Kupferlösung benutzt werden.

Diese Methode, für grössere Versuchsreihen besonders geeignet, liefert bei nur einiger Vorsicht die genauesten Resultate. Nur darf die Kupferlösung bei ihrem Gebrauch nicht allzusehr verdünnt werden; noch weniger darf man sich durch das scheinbare Entfärbtsein der Flüssigkeit verleiten lassen, die völlige Reduction des Kupferoxyd annehmen zu wollen. Der Punkt, wo alles Kupferoxyd reducirt ist, kann durch das Auge eines Geübten wohl annähèrnd, nie aber auf 1 bis $1 / 2$ p. C. bestimmt werden. Sehr leicht und mit grosser Schärfe findet man den Punkt der vollkommenen Reducirung des Kupferoxyd mittelst Kaliumeisencyanür, indem man zu der, durch ein doppeltes Filter filtrirten alkalischen Probe einen Tropfen einer sehr verdünnten Kaliumeisencyanürlösung setzt, und nun nach 
dem Umschütteln einige Tropfen einer sehr verdünnten Salzsäure ganz ruhig an den Wandungen des Probeglases hinabfliessen lässt, so dass die specifisch leichtere Salzsäure über die Probeflüssigkeit zu stehen kommt. Auf diese Weise wird sich auch die geringste Kupfermenge sogleich an den Berührungspunkten der beiden Flüssigkeiten durch die charakteristische rothbraune Farbe zu erkennen geben.

Säuert man die alkalische Lösung vor dem Zusatz von Kaliumeisencyanür an, so macht ein Tropfen Säure zu viel oder zu wenig die Reaction undeutlich oder gar nicht entstehen.

Die Bestimmung des Stickstoffs wurde nach der Methode von Peligot ausgeführt.

Die Bestimmung der Holzfaser und Pectin aus der Differenz berechnet.

\section{Analytische Belege. \\ I. Kalisalpeter.}

No. 1. 26,10 Grm. frische Substanz gaben bei $100^{\circ}$ getrocknet 3,164 Grm. trockne Substanz $=0,320$ Grm. Asche.

$1,045 \mathrm{Grm}$, trockne Substanz gaben $0,0346 \mathrm{NH}_{3}=$ 0,0285 Grm. Stickstoff.

No. 2. 26,61 Grm. fr. Sbstz. gaben 4,290 tr. Sbstz. $=\mathbf{0 , 3 1 5}$ Grm. Asche.

0,935 Grm. tr. Sbstz. gaben $0,0300 \mathrm{NH}_{3}=0,0247$ Grm. Stickstoff.

No. 3. 32,38 Grm. fr. Sbstz. gaben 4,712 tr. Sbstz. $=0,438$ Grm. Asche.

1,016 Grm. tr. Sbstz. gaben $0,0395 \mathrm{NH}_{3}=0,0325$ Grm. Stickstoff.

No. 4. 32,70 Grm. fr. Sbstz. gaben 4,810 tr. Sbstz. $=0,366$ Grm. Asche.

0,690 Grm. tr. Sbstz. gaben $0,0236 \mathrm{NH}_{3}=0,0194$ Grm. Stickstoff.

No. 5. 21,42 Grm. fr. Sbstz. gaben 3,080 tr. Sbstz. $=\mathbf{0 , 2 5 0}$ Grm. Asche. 
$0,740 \mathrm{Grm}$. tr. Sbstz. gaben $0,0306 \mathrm{NH}_{3}=0,0252$ Grm. Stickstoff.

No. 6. $25,90 \mathrm{Grm}$. fr. Sbstz. gaben 2,990 tr. Sbstz. $=0,310$ Grm. Asche.

\section{Holzasche.}

No. 1. 24,12 Grm. fr. Sbstz. gaben 3,480 tr. Sbstz. $=0,360$ Grm. Asche.

0,545 Grm. tr. Sbstz. gaben $0,0268 \mathrm{NH}_{3}=0,0221$ Grm. Stickstoff.

No. 2. 20,28 Grm. fr. Sbstz. gaben 2,280 tr. Sbstz. $=0,285$ Grm. Asche.

0,875 Grm. tr. Sbstz. gaben $0,0331 \mathrm{NH}_{3}=0,0272$ Grm. Stickstoff.

No. 3. 20,61 Grm. fr. Sbstz. gaben 2,850 tr. Sbstz. $=\mathbf{0 , 2 9 4}$ Grm. Asche.

$0,580 \mathrm{Grm}$. tr. Sbstz. gaben $0,0226 \mathrm{NH}_{3}=0,0168$ Grm. Stickstoff.

No. 4. 20,54 Grm. fr. Sbst. gaben 2,780 tr. Sbstz. $=0,260$ Grm. Asche.

$0,530 \mathrm{Grm}$. tr. Sbstz. gaben $0,0204 \mathrm{NH}_{3}=0,0168$ Grm. Sticksteff.

No. 5. 17,90 Grm. fr. Sbstz. gaben 1,980 tr. Sbstz. $=0,260$ Grm. Asche.

0,550 Grm. tr. Sbstz. gaben 0,0299 $\mathrm{NH}_{3}=0,0246$ Grm. Stickstoff.

No. 6. 24,45 Grm. fr. Sbstz. gaben 2,580 tr Sbstz. $=0,387$ Grm. Asche.

0,513 Grm. tr. Sbstz. gaben $0,0255 \mathrm{NH}_{3}=0,0210$ Grm. Stickstoff.

\section{Chlorammonium.}

No. 1. 20,56 Grm. fr. Sbstz. gaben 3,240 tr. Sbstz $=0,219$ Grm. Asche.

0,655 Grm. tr. Sbstz. gaben $0,0268 \mathrm{NH}_{3}=0,0221$ Grm. Stickstoff.

No. 2. 21,96 Grm. fr. Sbstz. gaben 3,413 tr. Sbstz. $=0,300$ Grm. Asche. 
0,573 Grm. tr. Sbstz. gaben $0,0140 \mathrm{NH}_{3}=0,0110$ Grm. Stickstoff.

No. 3. 21,12 Grm. fr. Sbstz. gaben 2,983 tr. Sbstz. $=0,312$ Grm. Asche.

1,233 Grm. tr. Sbstz. gaben 0,0446 $\mathrm{NH}_{3}=0,0367$ Grm. Stickstoff.

No. 4. $25,50 \mathrm{Grm}$. fr. Sbstz. gaben 3,799 tr. Sbstz. $=0,330$ Grm. Asche.

1,038 Grm. tr. Sbstz. gaben $0,0383 \mathrm{NH}_{3}=0,0315$ Grm. Stickstoff.

No. 5. 22,38 Grm. fr. Sbstz. gaben 2,986 tr. Sbstz. $=0,354$ Grm. Asche.

1,080 Grm. tr. Sbstz. gaben $0,0395 \mathrm{NH}_{3}=0,0325$ Grm. Stickstoff.

No. 6. 22,86 Grm. fr. Sbstz. gaben 3,123 tr. Sbstz. $=0,327$ Grm. Asche.

$1,020 \mathrm{Grm}$. tr. Sbstz. gaben $0,395 \mathrm{NH}_{3}=0,0325$ Grm. Stickstoff.

IV. Kochsalz.

No. 1. 19,50 Grm. fr. Sbstz. gaben 2,865 tr. Sbstz. $=0,180$ Grm. Asche.

1,105 Grm. tr. Sbstz. gaben $0,0299 \mathrm{NH}_{3}=0,0246$ Grm. Stickstoff.

No. 2. $23,73 \mathrm{Grm}$. fr. Sbstz. gaben 3,255 tr. Sbstz. $=0,345$ Grm. Asche.

1,470 Grm. tr. Sbstz. gaben $0,0497 \mathrm{NH}_{3}=0,0404$ Grm. Stickstoff.

No. 3. 19,68 Grm. fr. Sbstz. gaben 2,754 tr. Sbstz. $=0,210$ Grm. $\Lambda$ sche.

1,395 Grm. tr. Sbstz. gaben $0,0462 \mathrm{NH}_{3}=0,0380$ Grm. Stickstoff.

No. 4. 27,40 Grm. fr. Sbstz. gaben 2,240 tr. Sbstz. $=0,408$ Grm. Asche.

0,950 Grm. tr. Sbstz. gaben $0,0470 \mathrm{NH}_{3}=0,0387$ Grm. Stickstoff.

No. 5. Fehlt.

No. 6. 19,35 Grm. fr. Sbstz. gaben 2,019 tr. Sbstz. $=0,309$ Grm. Asche. 


\section{Soda.}

No. 1. 15,92 Grm. fr. Sbstz. gaben 2,286 tr. Sbstz. $=0,225$ Grm. Asche.

0,966 Grm. tr. Sbstz. gaben $0,0396 \mathrm{NH}_{3}=0,0326$ Grm. Stickstoff.

No. 2. Fehit.

No. 3. 20,31 Grm. fr. Sbstz. gaben 2,123 tr. Sbstz. $=0,288$ Grm: Asche.

1,080 Grm. tr. Sbstz. gaben $0,0351 \mathrm{NH}_{3}=0,0288$ Grm. Stickstoff.

No, 4. 23,04 Grm. fr. Sbstz. gaben 2,916 tr. Sbstz. $=0,363$ Grm. Asche.

1,093 Grm. tr. Sbstz. gaben $0,0299 \mathrm{NH}_{3}=0,0246$ Grm. Stickstoff.

No. 5. 24,75 Grm. fr. Sbstz. gaben $2,145 \mathrm{tr}$. Sbstz. $=\mathbf{0 , 3 9 0}$ Grm. Asche.

0,805 Grm. tr. Sbstz. gaben $0,0332 \mathrm{NH}_{3}=0,0273$ Grm. Stickstoff.

No. 6. 25,03 Grm. fr. Sbstz. gaben 4,050 tr. Sbstz. $=\mathbf{0 , 2 8 5}$ Grm. Asche.

0,760 Grm. tr. Sbstz. gaben 0,0312 $\mathrm{NH}_{3}=0,0257$ Grm. Stickstoff.

VI. Schwefelsaures Ammoniak.

No. 1. 19,48 Grm. fr. Sbstz, gaben 3,824 tr. Sbstz. $=0,204$ Grm. Asche.

0,961 Grm. tr. Sbstz. gaben 0,0268 $\mathrm{NH}_{3}=0,0223$ Grm. Sticksstoff.

No. 2. 22,52 Grm. fr. Sbstz. gaben 3,884 tr. Sbstz. $=0,252$ Grm. Asche.

0,880 Grm. tr. Sbstz. gaben $0,0363 \mathrm{NH}_{3}=0,0299$ Grm. Stickstoff.

No, 3. 21,74 Grm. fr. Sbstz. gaben 3,060 tr. Sbstz. $=0,264$ Grm. Asche.

$1,010 \mathrm{Grm}$. tr. Sbstz. gaben $0,373 \mathrm{NH}_{3}=0,0307$ Grm. Stickstoff.

No. 4. 16,68 Grm. fr. Sbstz. gaben 3,080 tr. Sbstz. $=0,272$ Grm. Asche. 
1,480 Grm. tr. Sbstz. gaben $0,0523 \mathrm{NH}_{3}=0,0430$ Grm. Stickstoff.

No. 5. 27,28 Grm. fr. Sbstz. gaben 3,608 tr. Sbstz. $=0,440$ Grm. Asche.

0,965 Grm. tr. Sbstz. gaben $0,0427 \mathrm{NH}_{3}=0,0351$ Grm. Stickstoff.

\section{Kohlensaures Ammoniak.}

No. 1. 24,52 Grm. fr. Sbstz. gaben 3,140 tr. Sbstz. $=0,266$ Grm. Asche.

0,722 Grm. tr. Sbstz. gaben $0,0331 \mathrm{NH}_{3}=0,0272$ Grm. Stickstoff.

No. 2. 21,54 Grm. fr. Sbstz. gaben 2,724 tr. Sbstz. $=0,320$ Grm. Asche.

0,795 Grm. tr. Sbstz. gaben $0,0332 \mathrm{NH}_{3}=0,0273$ Grm. Stickstoff.

No. 3. $24,08 \mathrm{Grm}$. fr. Sbstz. gaben 3,044 tr. Sbstz. $=0,380$ Grm. Asche.

0,885 Grm. tr. Sbstz. gaben $0,0427 \mathrm{NH}_{3}=0,0351$ Grm. Stickstoff.

No. 4. 20,68 Grm. fr. Shstz. gaben 2,868 tr. Sbstz. $=0,312$ Grm. Asche.

$1,370 \mathrm{Grm}$. tr. Sbstz. gaben $0,0468 \mathrm{NH}_{3}=0,0385$ Grm. Stickstoff.

No. 5. 20,72 Grm. fr. Sbstz. gaben 3,380 tr. Sbstz. $=0,300$ Grm. Asche.

$-1,230$ Grm. tr. Sbstz. gaben $0,0429 \mathrm{NH}_{3}=0,0353$ Grm. Stickstoff.

\section{Natronsalpeter.}

No. 1. 19,98 Grm. fr. Sbstz. gaben 3,360 tr. Sbstz. $=0,249$ Grm. Asche.

1,060 Grm. tr. Sbstz. gaben 0,0427 $\mathrm{NH}_{3}=0,0351$ Grm. Stickstoff.

No. 2. 25,00 Grm. fr. Sbstz. gaben 3,800 tr. Sbstz. $=0,320$ Grm. Asche.

$1,215 \mathrm{Grm}$. tr. Sbstz. gaben $0,0455 \mathrm{NH}_{3}=0,0374$ Grm. Stickstoff. 
No. 3. 20,37 Grm. fr. Sbstz. gaben 2,880 tr. Sbstz. $=0,279$ Grm. Asche.

1,491 Grm. tr. Sbstz. gaben 0,0427 $\mathrm{NH}_{3}=0,0351$ Grm. Stickstoff.

No. 4. 31,64 Grm. fr. Sbstz. gaben 3,800 tr. Sbstz. $=0,384$ Grm. Asche.

1,182 Grm. tr. Sbstz. gaben $0,0394 \mathrm{NH}_{3}=0,0324$ Grm. Stickstoff.

No. 5. 24,92 Grm. fr. Sbstz. gaben 3,740 tr. Sbstz. $=0,260$ Grm. Asche.

$1,200 \mathrm{Grm}$. tr. Sbstz. gaben $0,0331 \mathrm{NH}_{3}=0,0272$ Grm. Stickstoff.

\section{Pottasche.}

No. 1. 31,08 Grm. fr. Sbstz. gaben 4,400 tr. Sbstz. $=0,268$ Grm. Asche.

1,372 Grm. tr. Sbstz. gaben 0,0466 $\mathrm{NH}_{3}=0,0383$ Grm. Stickstoff.

No. 2. $27,28 \mathrm{Grm}$. fr. Sbstz. gaben 3,804 tr. Sbstz. $=0,276$ Grm. Asche.

$1,020 \mathrm{Grm}$. tr. Sbstz. gaben $0,0331 \mathrm{NH}_{3}=0,0272$ Grm. Stickstoff.

No. 3. 31,88 Grm. fr. Sbstz. gaben 5,004 tr. Sbstz. $=0,440$ Grm. Asche.

1,095 Grm. tr. Sbstz. gaben $0,0342 \mathrm{NH}_{3}=0,0281$ Grm. Stickstoff.

No. 4. $35,96 \mathrm{Grm}$. fr. Sbstz. gaben $5,660 \mathrm{tr}$. Sbstz. $=0,442$ Grm. Asche.

1,370 Grm. tr. Sbstz. gaben $0,0465 \mathrm{NH}_{3}=0,0383$ Grm. Stickstoff.

No. 5. 34,87 Grm. fr. Sbstz, gaben 5,524 tr, Sbstz. $=0,448$ Grm. Asche.

1,371 Grm. tr. Sbstz. gaben $0,0395 \mathrm{NH}_{3}=0,0325$ Grm. Stickstoff.

\section{Knochen.}

No. 1. 22,11 Grm. fr. Sbstz. gaben 3,524 tr. Sbstz. $=0,244$ Grm. Asche. 
1,071 Grm. tr. Sbstz. gaben $0,0331 \mathrm{NH}_{3}=0,0272$ Grm. Stickstoff.

No. 2. 27,84 Grm. fr. Sbstz. gaben 4,440 tr. Sbstz. $=0,420$ Grm. Asche.

0,945 Grm. tr. Sbstz. gaben $0,0268 \mathrm{NH}_{3}=0,0221$ Grm. Stickstoff.

No. 3. 38,88 Grm. fr. Sbstz. gaben 4,120 tr. Sbstz. $=\mathbf{0 , 5 4 0}$ Grm. Asche.

$0,936 \mathrm{Grm}$. tr. Sbstz. gaben $0,0265 \mathrm{NH}_{3}=0,0218$ Grm. Stickstoff.

No. 4. 27,52 Grm. fr. Sbstz. gaben 3,700 tr. Sbstz. $=0,340$ Grm. Asche.

10,52 Grm. tr. Sbstz. gaben $0,0329 \mathrm{NH}_{3}=0,0276$ Grm. Stickstoff.

No. 5. 22,76 Grm. fr. Sbstz. gaben 3,484 tr. Sbstz. $=0,280$ Grm. Asche.

1,035 Grm. tr. Sbstz. gaben $0,0236 \mathrm{NH}_{3}=0,0194$ Grm. Stickstoff.

\section{Kalk.}

No. 1. $17,90 \mathrm{Grm}$. fr. Sbstz. gaben $2,203 \mathrm{tr}$. Sbstz. $=0,231$ Grm. Asche.

No. 2. $23,68 \mathrm{Grm}$. fr. Sbstz. gaben 3,860 tr. Sbstz. $=\mathbf{0 , 3 6 0}$ Grm. Asche.

0,897 Grm. tr. Sbstz. gaben $0,0236 \mathrm{NH}_{3}=0,0194$ Grm. Stickstoff.

No. 3. 24,48 Grm. fr. Sbstz. gaben 3,204 tr. Sbstz. $=0,380$ Grm. Asche.

0,812 Grm. tr. Sbstz. gaben $0,0204 \mathrm{NH}_{3}=0,0168$ Grm. Stickstoff.

No. 4. 29,98 Grm. fr. Sbstz. gaben 4,140 tr. Sbstz. $=0,384$ Grm. Asche.

0,708 Grm. tr. Sbstz. gaben $0,0191 \mathrm{NH}_{3}=0,0157$ Grm. Stickstoff.

No. 5. 25,64 Grm. fr. Sbstz. gaben 2,888 tr. Sbstz. $=0,364$ Grm. Asche.

0,855 Grm. tr. Sbstz. gaben $0,0204 \mathrm{NH}_{3}=0,0168$ Grm. Stickstoff. 


\section{Gyps.}

No. 1. Fehlt.

No. 2. 24,28 Grm. fr. Sbstz. gaben 3,364 tr. Sbstz. $=0,280$ Grm. Asche.

0,930 Grm. tr. Sbstz. gaben $0,0195 \mathrm{NH}_{3}=0,0160$ Grm. Stickstoff.

No. 3. 16,98 Grm. fr. Sbstz. gaben 2,586 tr. Sbstz. $=0,207$ Grm. Asche.

0,957 Grm. tr. Sbstz. gaben $0,0186 \mathrm{NH}_{3}=0,0162$ Grm. Stickstoff.

No. 4. 31,20 Grm. fr. Sbstz. gaben 4,361 tr. Sbstz. $=0,367$ Grm. Asche.

$1,167 \mathrm{Grm}$. tr. Sbstz. gaben $0,0195 \mathrm{NH}_{3}=0,0161$ Grm. Stickstoff.

No. 5. 22,35 Grm. fr. Sbstz. gaben 3,423 tr. Sbstz. $=0,189$ Grm. Asche.

$1,290 \mathrm{Grm}$. tr. Sbstz. gaben $0,0280 \mathrm{NH}_{3}=0,0230$ Grm. Stickstoff.

\section{Animalischer Dünger.}

No. 1. 17,03 Grm. fr. Sbstz. gaben 2,325 tr. Sbstz. $=0,210$ Grm. Asche.

0,813 Grm. tr. Sbstz. gaben $0,0299 \mathrm{NH}_{3}=0,0246$ Grm. Stickstoff.

No. 2. $15,15 \mathrm{Grm}$. fr. Sbstz. gaben $2,493 \mathrm{tr}$. Sbstz. $=0,228$ Grm. Asche.

0,813 Grm. tr. Sbstz. gaben $0,0292 \mathrm{NH}_{3}=0,0245$ Grm. Stickstoff.

No. 3. 24,28 Grm. fr. Sbstz. gaben 4,240 tr. Sbstz. $=0,204$ Grm. Asche.

0,734 Grm. tr. Sbstz. gaben $0,0280 \mathrm{NH}_{3}=0,0236$ Grm. Stickstoff.

No. 4. 28,80 Grm. fr. Sbstz. gaben 3,456 tr. Sbstz. $=0,426$ Grm. Asche.

0,960 Grm. tr. Sbstz. gaben $0,312 \mathrm{NH}_{3}=0,0257$ Grm. Stickstoff. 
Aus diesen Ergebnissen berechnet sich die Zusammensetzung der frischen Rüben auf $\mathbf{1 0 0}$ Theile:

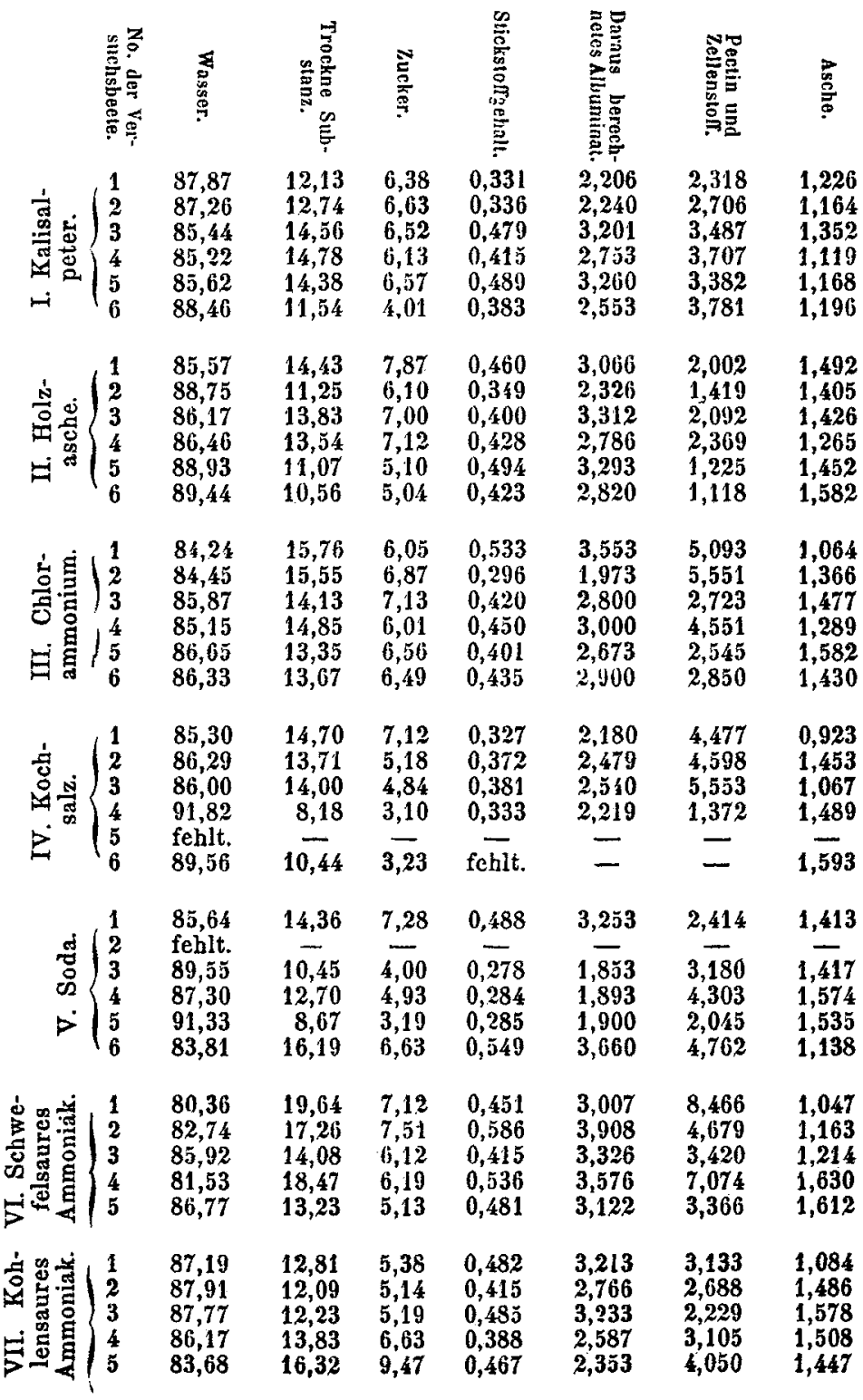


auf die Entwicklung der Zuckerrübe.

\begin{tabular}{|c|c|c|c|c|c|c|c|c|}
\hline & 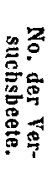 & 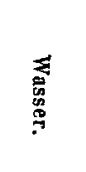 & 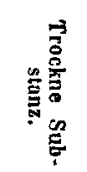 & 容 & 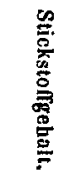 & 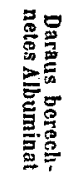 & 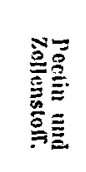 & $\begin{array}{l}\text { D. } \\
\frac{0}{0}\end{array}$ \\
\hline 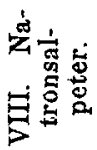 & $\left\{\begin{array}{l}1 \\
2 \\
3 \\
4 \\
5\end{array}\right.$ & $\begin{array}{l}83,18 \\
84,80 \\
85,86 \\
87,99 \\
84,99\end{array}$ & $\begin{array}{l}16,82 \\
15,20 \\
14,14 \\
12,01 \\
15,01\end{array}$ & $\begin{array}{l}9,01 \\
8,10 \\
7,07 \\
6,20 \\
8,93\end{array}$ & $\begin{array}{l}0,572 \\
0,467 \\
0,333 \\
0,329 \\
0,340\end{array}$ & $\begin{array}{l}3,812 \\
3,113 \\
2,220 \\
2,193 \\
2,266\end{array}$ & $\begin{array}{l}2,752 \\
2,707 \\
3,481 \\
2,404 \\
2,771\end{array}$ & $\begin{array}{l}1,246 \\
1,280 \\
1,369 \\
1,213 \\
1,043\end{array}$ \\
\hline 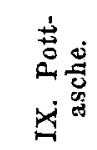 & $\left\{\begin{array}{l}1 \\
2 \\
3 \\
4 \\
5\end{array}\right.$ & $\begin{array}{l}85,84 \\
86,05 \\
84,30 \\
84,26 \\
84,15\end{array}$ & $\begin{array}{l}14,16 \\
13,95 \\
15,70 \\
15,74 \\
15,85\end{array}$ & $\begin{array}{l}7,12 \\
7,25 \\
9,20 \\
9,56 \\
7,41\end{array}$ & $\begin{array}{l}0,395 \\
0,371 \\
0,103 \\
0,396 \\
0,376\end{array}$ & $\begin{array}{l}2,633 \\
2,473 \\
2,686 \\
2,640 \\
2,506\end{array}$ & $\begin{array}{l}3,545 \\
3,216 \\
2,434 \\
2,311 \\
4,650\end{array}$ & $\begin{array}{l}0,862 \\
1,011 \\
1,380 \\
1,229 \\
1,284\end{array}$ \\
\hline 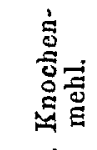 & $\left\{\begin{array}{l}1 \\
2 \\
3 \\
4 \\
5\end{array}\right.$ & $\begin{array}{l}84,06 \\
84,05 \\
89,40 \\
86,55 \\
85,13\end{array}$ & $\begin{array}{l}15,94 \\
15,95 \\
10,60 \\
13,45 \\
14,87\end{array}$ & $\begin{array}{l}7,41 \\
6,51 \\
5,73 \\
5,60 \\
6,88\end{array}$ & $\begin{array}{l}0,404 \\
0,379 \\
0,246 \\
0,346 \\
0,278\end{array}$ & $\begin{array}{l}\mathbf{2}, 693 \\
\mathbf{2}, \mathbf{5 2 6} \\
1,612 \\
2,308 \\
\mathbf{1 , 8 5 3}\end{array}$ & $\begin{array}{l}4,733 \\
5,405 \\
1,870 \\
4,307 \\
4,907\end{array}$ & $\begin{array}{l}1,104 \\
1,309 \\
1,388 \\
1,235 \\
1,230\end{array}$ \\
\hline 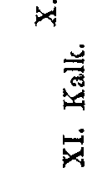 & $\begin{array}{l}1 \\
2 \\
3 \\
4 \\
5\end{array}$ & $\begin{array}{l}87,69 \\
83,69 \\
86,91 \\
86,19 \\
88,73\end{array}$ & $\begin{array}{l}12,31 \\
16,31 \\
13,09 \\
13,81 \\
11,27\end{array}$ & $\begin{array}{l}5,51 \\
6,00 \\
5,37 \\
5,30 \\
5,01\end{array}$ & $\begin{array}{l}\overline{0,352} \\
0,270 \\
0,306 \\
0,221\end{array}$ & $\begin{array}{l}2, \overline{347} \\
1,800 \\
2,400 \\
1,466\end{array}$ & $\begin{array}{l}\overline{6,443} \\
4,368 \\
4,830 \\
3,375\end{array}$ & $\begin{array}{l}1,290 \\
1,520 \\
1,552 \\
1,280 \\
1,419\end{array}$ \\
\hline 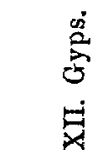 & $\begin{array}{l}1 \\
2 \\
3 \\
4 \\
5\end{array}$ & $\begin{array}{l}\text { fehlt. } \\
86,14 \\
84,80 \\
86,02 \\
84,68\end{array}$ & $\begin{array}{c}-\overline{13,86} \\
15,20 \\
13,98 \\
15,32\end{array}$ & $\begin{array}{l}-\overline{5,73} \\
6,57 \\
5,85 \\
5,96\end{array}$ & $\begin{array}{c}-\overline{0,238} \\
0,257 \\
0,192 \\
0,274\end{array}$ & $\begin{array}{l}\overrightarrow{1,586} \\
1,713 \\
1,218 \\
1,826\end{array}$ & $\begin{array}{l}5, \overline{391} \\
5,698 \\
5,736 \\
6,689\end{array}$ & $\begin{array}{l}-\overline{1,153} \\
1,219 \\
1,176 \\
0,845\end{array}$ \\
\hline 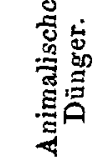 & $\begin{array}{l}1 \\
2 \\
3 \\
4\end{array}$ & $\begin{array}{l}86,36 \\
84,20 \\
82,57 \\
88,00\end{array}$ & $\begin{array}{l}13,64 \\
15,80 \\
17,43 \\
12,00\end{array}$ & $\begin{array}{l}6,91 \\
6,73 \\
6,85 \\
6,27\end{array}$ & $\begin{array}{l}0,413 \\
0,476 \\
0,560 \\
0,320\end{array}$ & $\begin{array}{l}2,753 \\
\mathbf{3}, 162 \\
\mathbf{3}, 733 \\
2,133\end{array}$ & $\begin{array}{l}2,741 \\
4,404 \\
6,007 \\
2,118\end{array}$ & $\begin{array}{l}1,233 \\
1,504 \\
0,840 \\
1,479\end{array}$ \\
\hline
\end{tabular}

\section{Kalisalpeter.}

Der Einfluss des Kalisalpeters ist auf den Ertrag ein günstiger, indem er denselben erhöhte. Auf den Zucker und Aschengehalt scheint er nicht influirt zu haben; dagegen ist der Stickstoffgehalt ein bedeutend erhöhter.

\section{Holzasche.}

Steigerte den Ertrag und übte in geringer Menge aufgebracht auf den Zuckergehalt einen günstigen Einflüss. Der Zuckergehalt nimmt nur mit steigender Salzmenge ab. 
Bei zunehmendem Aschengehalt, steigt auch der Stickstoffgehalt beträchtlich.

\section{Chlorammonium.}

Bei sehr hohem Ertrage scheint derselbe proportional mit zunehmender Salzmenge zu steigen. Der Zuckergehalt ist nicht verringert. Der Aschen- und Stickstoffgehalt ist ein sehr erhöhter.

\section{Kochsal*.}

Wenn man den Einfluss des Kochsalzes auf den Ertrag keinen ungünstigen nennen kann, so ist seine Wirkung auf den Zuckergehalt der Rübe ein entschieden nachtheiliger zu nennen. Der Zuckergehalt nimmt mit steigender Salzmenge ab. Der Aschengehalt ist erhöht - der Stickstoffgehalt dagegen vermindert.

\section{Soda.}

Uebte sowohl auf den Ertrag, als auch auf den Zuckergehalt einen entschieden nachtheiligen Einfluss aus. Sonst ist seine Wirkung dem Kochsalz gleich.

\section{Schwefelsaures Ammoniak.}

Der Einfluss dieses Salzes auf das Ernteergebniss ist im Allgemeinen ein geringerer, als dies bei den übrigen angewandten Ammoniaksalzen der Fall ist. Der Zuckergehalt scheint nicht vermindert, dagegen ist der Aschenund Stickstoffgehalt ein hoher zu nennen.

VII. Kohlensaures Ammoniak.

Erhöht den Ertrag, und verhält sich im übrigen wie das schwefelsaure Ammoniak.

\section{Natronsalpeter.}

Sehr in die Augen fallend ist der hohe Ertrag - der mit steigender Salzmenge zuzunehmen scheint. Der Zuckergehalt ist erhöht bei geringerem Aschengehalt und nicht allzugrosser Stickstoffmenge. 


\section{Pottasche.}

hat unter allen angewandten Salzen den höchsten Ertrag und grössten Zuckergehalt geliefert. Der Aschengehalt und die Stickstoffmenge sind nicht bedeutend hoch.

\section{Knochenmehl.}

Hat den Ertrag erhöht. Der Zuckergehalt und Stickstoffgehalt sind vermindert. Der Aschengehalt im Allgemeinen ein erhöhter.

\section{Kalk.}

Das Ernteergebniss ist ein bedeutendes. - Der Zuckergehalt und Stickstoffgehalt vermindert. Der Aschengehalt sehr hoch.

\section{Gyps.}

Verhält sich wie Kalk, nur ist die Aschenmenge nicht so hoch wie bei Kalk.

\section{Amimalischer Dünger.}

Bei hohem Ertrag ist die Zuckermenge eine verhältnissmässig geringe. Der Aschengehalt sehr schwankend. Der Stickstoffgehalt ein constant hoher.

Allgemeinere Schlüsse lassen sich, wie aus den Tabellen ersichtlich, aus der so sehr schwankenden $\mathrm{Zu}$ sammensetzung der Rüben ohne allzugrosse Willkürlichkeit nicht ziehen.

Gewisse Beziehungen zwischen dem Zucker- und Stickstoffgehalt ergeben sich weder aus der Analyse auf frische noch auf trockne Substanz berechnet, und es ist somit die Annahme : dass der Zuckergehalt mit steigender Stickstoffmenge fallt und umgekehrt, noch durch weitere Thatsachen zu bekräftigen, was nur durch zahlreich ausgeführte Analysen nach einer Methode erreicht werden dürfte - sobald es nämlich erwiesen ist - dass auch wirklich sämmtlicher in der Rübe enthaltener Stickstoff organisirter Stickstoff ist - welch letztere Annahme einige 
von mir vorläufig angestellte Versuche nicht zu bestätigen scheinen, indem sie mir die Gegenwart ron Ammoniaksalzen im frischen Rübensaft mehr als wahrscheinlich machen. Sollten weitere Versuche letztere Thatsache bestätigen, so müsste freilich zur Auffindung dieses Gesetzes eine andere Versuchsmethode, als die bisher übliche, eingehalten werden.

Ich habe in vorstehender Tabelle aus rein praktischem Interesse sämmtlichen durch die Analyse gefundenen Stickstoff als Albuminat berechnet, um dadurch den Faserstoff und das Pectin indirect bestimmen zu können.

Die Wässrigkeit der zur Analyse verwendeten Rüben mag eines Theils durch den regnerischen Sommer bedingt sein, wodurch natürlich die Menge der festen Bestandtheile ebenfalls etwas vermindert wurde. $\mathrm{Ob}$ jedoch der gegen andere Analysen auffallend geringe Zuckergehalt eben darin begründet sein soll, scheint mir mehr als zweifelhaft, im Gegentheil möchte man glauben, dass der Zuckergehalt der Rüben bisher im Allgemeinen zu hoch angenommen wurde. Nimmt man die Menge der festen Bestandtheile (wie dies auch aus den Untersuchungen von Schmitt*) hervorgeht, zu 13 bis $14 \mathrm{p}$. C. an, so ist neben dem Aschen-, Stickstoff-, Faserstoff- und Pectingehalt ein solcher Zuckergehalt von 10 bis 14 p. C. unerklärlich; und es dürfte ein Zuckergehalt von 7 p. C. als der normale zu betrachten sein.

Im Allgemeinen dürfte man aus der eben angeführten Tabelle ersehen, dass eine Zufuhr von anorganischen leicht löslichen Salzen den Aschengehalt vermehrt. Dass diese Vermehrung bei einigen z. B. bei Kali und Natronsalpeter etc. nicht ersichtlich ist, mag seinen Grund in der bei höheren Temperaturen so leichten Zersetzbarkeit der Salpetersäure haben, was man auch deutlich an den sich entwickelnden salpetrigen Dämpfen beim Verbrennen wahrnehmen konnte.

Bezüglich des Faserstoffs und Pectins haben dieselben

*) Annalen der Chemie und Pharmacie, Bd. LXXXIII, Heft 3. 
Măumené: Umwandlung d. Rohrz. durch Wasser. 147

bei den so häufigen Schwankungen nur dann Gültigkeit, wenn man allen Stickstoff als Albuminat zu berechnen berechtigt ist.

Da ich im Nächsten diese Versuche zu wiederholen gedenke, so dürften solche Versuche, wenn auch von Andern angestellt, gewiss bald zu einer rationellen Rübenkultur führen, welche allein diesem so wichtigen Industriezweige eine festere Grundlage $\mathrm{zu}$ sichern im Stande sind.

Heidelberg, im Januar 1855.

\title{
XXII. \\ Ueber die Umwandlung des Rohrzuckers durch Wasser.
}

\author{
Von
}

E. Maumené.

(Compt. rend. XXXIX, p. 914.)

Rohrzucker verwandelt sich unter dem Einflusse verdünnter Säuren, besonders beim Erwärmen, in unkrystallisirbaren, die Polarisationsebene nach links drehenden Zucker. Man schreibt allgemein den Säuren das ausschliessliche Vermögen $\mathrm{zu}$, diese Umwandlang, welche in der Vereinigung des Zuckers mit ein Aequivalent Wasser besteht, herbeizuführen; und man hat noch nicht hinreichend untersucht, welche Rolle das Wasser selbst bei diesem Processe spielt*). Ich habe diese Untersuchung vorgenommen, und, wie sich erwarten liess, gefunden, dass Wasser allein hinreicht, die in Rede stehende Umwandlung

*) Dumas sagt in seinem Traite de Chimie: Es genügt, Wasser 15 bis 20 Stunden im Sieden zu erhalten, um den Zucker, den es aufgelöst enthält, in Glucose und unkrystallisirbaren Zucker umzuwandeln. 